\title{
The Association of Disability and Pain with Individual and Community Socioeconomic Status in People with Hip Osteoarthritis
}

\author{
Joshua B. Knight, Leigh F. Callahan", My-Linh N. Luong, Jack Shreffler, Britta Schoster, \\ Jordan B. Renner and Joanne M. Jordan
}

\begin{abstract}
Thurston Arthritis Research Center and the Department of Medicine and Social Medicine, University of North Carolina at Chapel Hill, NC, USA
\end{abstract}

\begin{abstract}
Objective: To examine associations between disability and socioeconomic status (SES) in persons with hip radiographic OA (rOA) or symptomatic OA (sxOA) in the Johnston County Osteoarthritis Project.

Methods: Cross-sectional analyses were conducted on individuals with hip rOA (708) or sxOA (251). rOA was defined as Kellgren-Lawrence $\geq 2$. Educational attainment ( $<12$ years or $\geq 12$ years) and occupation (managerial or non-managerial) were individual SES measures. Census block group poverty rate $(<12 \%, 12-25 \%, \geq 25 \%)$ was the community SES measure. Disability was measured by the HAQ-DI and the WOMAC (function, pain, total). Covariates included age, gender, race, BMI, and presence of knee symptoms. Analyses examined associations of disability with each SES effect separately, followed by multivariable analyses using all SES variables, adjusting for covariates.

Results: In models with single SES variables adjusted for covariates, WOMAC scores were associated significantly $(\mathrm{p}<0.05)$ with low educational attainment and non-managerial occupation in rOA and sxOA. HAQ was significantly associated with low educational attainment in rOA and sxOA and with high community poverty in rOA. In models including all SES variables, the patterns of association were similar although with diminished significance. There was indication that education was more strongly associated with HAQ and WOMAC function, while occupation was more strongly associated with WOMAC pain.

Conclusion: Our data provide evidence that individual SES is an important factor to consider when examining disability and pain outcomes in older adults with hip OA.
\end{abstract}

Keywords: Disability, hip osteoarthritis, socioeconomic status, educational attainment, occupation, community poverty, pain.

\section{INTRODUCTION}

Arthritis, particularly osteoarthritis (OA), or joint pain of the lower extremities, can greatly restrict one's mobility in the workplace as well as hinder a variety of daily tasks at home [1]. OA in particular is responsible for roughly $20 \%$ of all arthritis-related ambulatory care visits [2], and results in \$3.4-13.2 billion in job-related costs each year [3]. Knee and hip replacements alone account for $35 \%$ of all arthritisrelated procedures requiring hospitalization [4].

In addition to important contributors to OA such as old age [3], high body mass index (BMI) [5], being female [3], and being African American [6, 7], an individual's socioeconomic status (SES) may have an effect on OA and any associated disability. A number of studies have linked physically-difficult, labor-intensive occupations to increased incidence of OA-related pain [8, 9] and OA-associated disability [10]. Also, lower levels of educational attainment are associated with the degree to which a person may be disabled [11, 12], but educational attainment is also a determinant of occupation selection, which can then increase predisposition to disability [13].

*Address correspondence to this author at the Thurston Arthritis Research Center, 3300 Thurston Building, CB 7280, University of North Carolina, Chapel Hill, NC 27599, USA; Tel: 919-966-0564; Fax: 919-966-1739;

E-mail: leigh_callahan@med.unc.edu
To our knowledge only a few studies have examined associations between educational attainment and disability and pain in hip OA. A study of 118 individuals from Finland with self-reported hip pain and radiographic hip OA (rOA) [14] found associations between disability measured by the WOMAC index and educational attainment. The 'EUROHIP' consortium [15] reported worse disability measured by the WOMAC associated with lower levels of educational attainment in a large cohort of persons with radiographic hip OA requiring surgical intervention [15]. And, in analyses of patients of the Dresden Hip Surgery Registry, the risk of non-response to hip surgery when measured by the WOMAC decreased with increasing educational attainment [16].

The socioeconomic characteristics of a community may also affect a person's predisposition to disability associated with OA. Persons with OA in communities with higher percentages of poverty are more likely to have less overall access to healthcare [17], higher percentages of confounding pre-existing conditions such as diabetes [18], higher rates of depression [1], or poor perception of quality of life [19]. Studies in the United Kingdom have found correlations between knee pain and physical aspects of the community environment, such as poor access to public transportation [20], and similar studies in the United States have found that persons with or at risk for symptomatic knee OA felt that 
their daily activities were limited due to poor transportation access or other barriers to their mobility [21]. Studies in the UK have found links between self-reported, non-specific disabling pain and a number of area-level, or community deprivation indicators including poverty, housing quality, and community layout [1]. No work, however, has been done to examine associations between quantitatively-evaluated disability and community-level SES indicators in individuals specifically with OA of the hip.

The purpose of this investigation is to explore the relationship of disability and pain outcomes to individual SES measures of education and occupation, and to community poverty level in persons exhibiting the two commonly used definitions of OA: hip radiographic OA (rOA) or hip symptomatic OA, the latter defined as having both radiographic evidence of $\mathrm{OA}$ and symptoms in the same joint. The study will also examine whether individual and community SES are independently associated with disability.

\section{MATERIALS AND METHODOLOGY}

The data used in this investigation were derived from the Johnston County Osteoarthritis (OA) Project (JoCo OA). This study is an ongoing, longitudinal, population-based study of knee and hip OA, including both rural and urban areas of Johnston County, North Carolina. Details on the recruitment of participants and overall project design are described in other literature [22]. The members of the population studied were non-Hispanic, English-speaking Caucasian or African American men and women, 45-years or older who were residents of one of six townships in the county for at least one year and who were mentally and physically capable of completing the study protocol. Each participant was interviewed at home, underwent a clinical exam and radiographs at the study center, and then was interviewed again at home approximately two weeks after the clinical exam.

In this paper, we selected data from baseline participants (i.e. those who enrolled in JoCo OA from May 1991 through December 1997) who returned for follow-up in 1999-2004 $(n=1858)$ or who were newly-enrolled during cohort enrichment in 2003-2004 $(n=1150)$. The cohort enrichment sample included younger individuals, men and African American individuals who were lost in greater proportion than other participants over the follow-up period from study initiation [23].

A total of 3008 participants were available and 2425 had complete data on hip rOA, HAQ or WOMAC scores, SES variables and covariates used in models (Fig. 1). The study parameters were approved by the Institutional Review Boards of both the Centers for Disease Control and Prevention as well as the School of Medicine at the University of North Carolina.

Data from participants' interviews and examinations in this large population screening were used to define a sample with radiographic OA (rOA) and a subgroup of this sample with symptomatic OA. We used both definitions since they are the two most commonly used.

Radiographically confirmed knee $O A$ (rOA). Radiographs were obtained during clinical exams. Radiographs of the hip were supine anterior-posterior pelvic radiographs with legs in $15^{\circ}$ rotation and interpreted by one radiologist (JBR) who scored for OA on the Kellgren/Lawrence $(\mathrm{K} / \mathrm{L})$ scale from 0 to 4 [24]. A score of 0 represents normal; a score of 1 is of questionable significance; and scores of 2 to 4 represent mild, moderate, and severe joint narrowing, respectively, due to OA [24]. For this investigation, radiographic OA was defined as $\mathrm{K} / \mathrm{L} \geq 2$ in at least one joint. Another radiologist was employed to examine interrater reliability; both interand intrarater reliability for the study radiologist were high (weighted inter-rater reliability $=0.86 ; \kappa$ for intra-rater reliability $=0.89$ ) [25]. The clinical exam also included measures of height and current weight to calculate BMI (weight in kilograms/[height in meters] $]^{2}$ ).

Total participants at the Johnston Counry OA Project first follow-up (1990-2003) and new enrollees (2003-2004)

\section{$n=3008$}

examination and

household interviews

$n=2425$
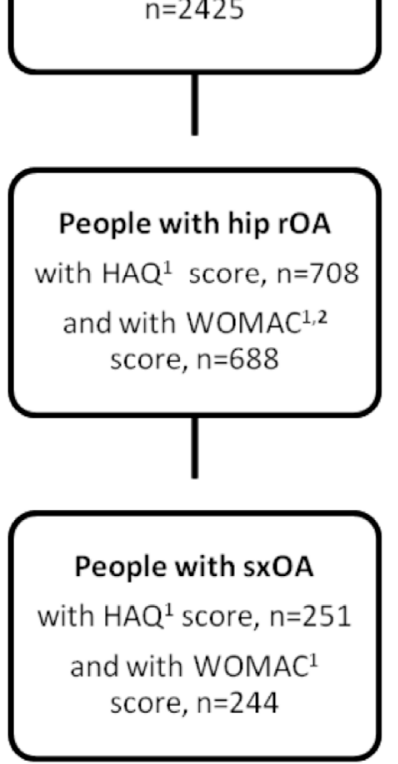

$1 \mathrm{HAQ}=$ Health Assessment Questionnaire, WOMAC = Western Ontario McMaster Universities Osteoarthritis Index

${ }^{2}$ WOMAC scores were collected at participants' clinical examination. Due to time constraints of the clinical examination, slightly fewer respondents have WOMAC scores collected.

${ }^{3}$ sXOA = symptomatic $O A$

Fig. (1). Participant flow chart of Johnston County Osteoarthritis (OA) Project participants at first follow-up.

Symptomatically confirmed knee $O A$. All participants completed symptom questionnaires as part of their in-home interview. A diagnosis of symptomatic osteoarthritis was made if the participant presented with $\mathrm{rOA}(\mathrm{K} / \mathrm{L}$ grade $\geq 2$ in at least one joint) and had an affirmative answer to the question, "on most days, do you have pain, aching, or stiffness in your left hip [right hip]?" This was asked 
separately with regard to each hip. Answers were coded into three categories; $0, \mathrm{rOA}$ without symptoms; 1 , $\mathrm{rOA}$ with mild symptoms; and 2, rOA with moderate or severe symptoms.

Additional information was collected from each participant during a home interview in the form of a questionnaire, and included questions on the participants' age, gender, race, highest education level and occupation. Based on their longest held job, participants were also asked to report their daily occupational activities (standing, walking, squatting, and lifting) and the frequency of each activity was assigned a number: $0=$ never, $1=$ seldom, $2=$ sometimes, $3=$ often, and $4=$ always. The numerical responses for each of the activities were then summed to produce an occupational physical activity score (PAS) that ranged from 0 to 16 [26].

Outcomes: Disability and Pain measures. A selfreported functional status assessment, the Stanford Health Assessment Questionnaire Disability Index (HAQ-DI or HAQ), was administered to each participant; the HAQ covers 20 activities of daily living sorted across eight domains (dressing, arising, eating, walking, reaching, gripping, chores, and hygiene) [27] to assess disability over the past week. Each activity is scored on a scale from 0 to 3, 0 meaning no difficulty, 1 meaning with little difficulty, 2 meaning with much difficulty, and 3 meaning unable to perform. The maximum scores from each of the eight domains were averaged together to produce the HAQ score (0-3) with a higher HAQ score implying more disability. In addition, participants answered the Western Ontario and McMaster Universities Index of Osteoarthritis (WOMAC) questionnaire, a validated self-administered instrument designed to evaluate knee and hip osteoarthritis. Our study asked participants to answer questions based on a two day recall period. The WOMAC includes subscales related to physical function, stiffness, and pain, with 17,2 , and 5 questions, respectively. When asked about the difficulty of performing tasks and degree of stiffness or pain, answers were rated on an ordinal scale of 0 to 4 , with lower levels indicating lower levels of symptoms or physical disability (i.e. $0=$ none, $1=$ mild, $2=$ moderate, $3=$ severe, and $4=$ extreme). In this analysis, we use the unweighted WOMAC, the total score (0-96), the physical function score (0-68), and the pain score (0-20). Higher scores on the WOMAC indicate increased pain, increased stiffness, and decreased physical function [28]. The items of the physical function subscale have similarities to $\mathrm{HAQ}$ and contribute a major portion of the total score [29]. Total WOMAC scores have demonstrated reliability and validity [30]. However, despite the good internal consistency of the WOMAC stiffness subscale, evidence supporting the use of the stiffness subscale is sparse; previous literature has shown that test-retest is low and convergent validity for hip patients is sparse [31]. For this reason, the WOMAC stiffness score is not included in the analysis.

Independent Variables. The primary exposure variables are three measures of SES: educational attainment, occupation, and community household poverty rate.

Educational Attainment. Participants were asked about their highest school year completed. They were then grouped into one of two categories, having less than 12 years or having 12 or more years of schooling [referent].

Occupation. Based on U.S. Census classifications, participants were grouped by one of seven occupational groups: (1) farming, forestry, or fishing; (2) management or professional; (3) fabrication or manual labor; (4) precision production, crafting, and repair; (5) service; (6) technology, sales, or administration; and (7) military [32]. For this investigation, groups $1,3,4,5$, and 7 were placed into a "non-managerial" occupational category, and groups 2 and 6 were placed into a "managerial" [referent] category. Occupational category is based on the job held for the longest period of time in the participant's life. The occupation of the longest job held has been demonstrated to be correlated with one's current occupation [33]. These two categories were then represented by an indicator variable in the analysis. "Managerial" is not to be taken entirely literally, but rather implies occupations that tend to include office work, lower physical demand, and higher SES, based on the Census group descriptions.

Community Poverty Level. To determine community poverty level, we used household income data at the block group level as provided from the U.S. 2000 Census [34, 35]. The census block group averages about 1000 residents, but can vary considerably, and is taken here to represent the participants' immediate community or neighborhood. The physical address of each participant at the time of evaluation was geocoded, linked to a block group identification number, and then used to extract aggregated census information from the U.S. 2000 Census. The percentage of households in each block group below the poverty level as defined by U.S. Census Bureau poverty thresholds was calculated and poverty level variables were categorized into tertiles (low, medium, and high) with cutpoints at $12 \%$ and $25 \%$. Individuals living in block groups where less than $12 \%$ were at the poverty level were categorized as living in a block group with "low" community poverty [referent]. Individuals living in block groups with greater than $25 \%$ at the poverty level were categorized as living in a block group with "high" community poverty. Individuals between $12 \%$ and $25 \%$ were categorized as "medium." There were equivalent distributions for low and high ( $25 \%$ in each of those groups) and a larger middle group (50\%) to reflect the mid-range of community poverty. Each participant's community poverty classification is determined by the block group in which they reside and not by their individual circumstances (i.e. residents of a block group share the same community household poverty rate). About $18 \%$ of the participants were in the high poverty group, and $27 \%$ were in the low poverty group.

Covariates. Covariates were age group indicators (45-54 years [referent], 55-64 years, $\geq 65$ years), gender (male[referent]/female), an obesity indicator based on BMI $\left(<30 \mathrm{~kg} / \mathrm{m}^{2}\right.$ [referent], $\left.\geq 30 \mathrm{~kg} / \mathrm{m}^{2}\right)$, race (Caucasian [referent] or African American), occupational physical activity score (PAS) $(<10$ [referent], $\geq 10)$, a count of non-musculoskeletal comorbidities, and indicators for presence of knee symptoms (yes/no [referent]), self-reported in the same manner as hip symptoms. Knee symptoms could play a large role in disability and must be controlled for. PAS was included as it directly detects occupational demands on the lower body 
rather than representing SES. The comorbidity count was determined on 11 possible conditions, with the most frequent being high blood pressure (49\%), chronic lung disease including asthma $(20 \%)$, diabetes $(17 \%)$, and circulatory problems or stroke $(14 \%)$.

\section{Statistical Analyses}

Statistical analysis was completed in STATA version 9.2. Educational attainment and occupation represented the main variables for individual SES. The block group poverty level is used for the main community SES variable as it represents a general sense of the community economic well-being, apart from the individual situation.

The characteristics of the participants and the analyses using single SES variables are based only on the participants who are eligible to be included in the fully-adjusted modeling of HAQ with all main effects, which included 708 with rOA and 251 with symptomatic. Not all participants completed the WOMAC due to time constraints at the clinical visit where it was collected, leaving 688 with radiographic OA and 244 with symptomatic OA (Fig. 1).

Within the hip rOA group and the hip symptomatic subgroup, associations between the SES variables and each of the four disability scores, as dependent variables, were calculated by ordinary linear regression. Both the HAQ and the WOMAC distributions are skewed to the right, with relatively high numbers of low values, and resulting distributions of residuals are asymmetric and not improved through transformation. Although linear regression tends to be robust against underlying assumptions, we performed additional analyses using proportional odds models with disability variables in five ordered classes, after first verifying the appropriateness of the approach versus polytomous logistic models. The findings of significance were highly similar to those for ordinary regression, as has previously been noted for WOMAC [36]. Linear regression is retained for ease in interpreting results. Preliminary regressions involved one of the three SES variables at a time, adjusted for age, gender, race, BMI, knee symptoms, comorbidity count, and occupational PAS. The final multivariable regressions included all three SES variables and the covariates simultaneously and indicated whether the SES variables were independently associated with the outcomes. Adjusted parameter estimates and 95\% confidence intervals are reported in the tables.

\section{RESULTS}

Participants. The characteristics of the sample population for hip rOA and subgroup with symptomatic OA are presented in Table 1. The mean age of individuals with hip rOA or symptomatic OA was 67 . The mean disability scores were higher in the symptomatic $\mathrm{OA}$ subgroup $(\mathrm{HAQ}=0.82$; WOMAC total $=33.7)$ than $\mathrm{rOA}(\mathrm{HAQ}=0.52$; WOMAC total=22.4), indicating worse disability. The higher WOM $\mathrm{AC}$ total score was reflected in both the pain and function subscales. Fifty percent of the individuals with hip symptomatic OA and $43 \%$ of those with radiographic OA had a BMI greater than 30 . Compared to those with hip rOA, those in the subgroup with symptomatic hip OA were more likely to have low educational attainment and a non-managerial occupation, but had roughly comparable community poverty rates.

Table 1. Demographic, Clinical, Socioeconomic, and Disability Characteristics of the Participants who have Radiographic Hip $O A$ and the Subgroup with Symptomatic Hip OA

\begin{tabular}{|c|c|c|}
\hline Variable & $\begin{array}{c}\text { Radiographic } \\
\text { Hip OA }(N=708)\end{array}$ & $\begin{array}{c}\text { Symptomatic } \\
\text { Hip OA }(\mathrm{N}=251)\end{array}$ \\
\hline & \multicolumn{2}{|c|}{ Mean (sd) or Percent } \\
\hline Age, $y$ & $67.0(10.3)$ & $66.7(10.2)$ \\
\hline $45-54 y$ & 14.8 & 14.3 \\
\hline $55-64 y$ & 30.8 & 33.1 \\
\hline$\geq 65 y$ & 54.4 & 52.6 \\
\hline BMI, $\mathrm{kg} / \mathrm{m} 2$ & $30.0(6.3)$ & $31.3(6.9)$ \\
\hline $\mathrm{BMI} \geq \mathbf{3 0}$ & 43.2 & 50.2 \\
\hline Female & 61.0 & 66.9 \\
\hline African American & 26.6 & 23.5 \\
\hline $\operatorname{PAS}^{3}(0-16)$ & $9.59(3.53)$ & $9.80(3.57)$ \\
\hline High $P A S^{3} \geq 10$ & 54.2 & 58.6 \\
\hline Knee Symptoms & 52.8 & 72.1 \\
\hline Comorbidity Count (0-11) & $1.37(1.28)$ & $1.70(1.48)$ \\
\hline Education, $<12 \mathrm{yr}$ & 32.9 & 37.1 \\
\hline Occupation, $\mathbf{N M}^{4}$ & 53.2 & 58.2 \\
\hline Poverty Rate & $18.4(10.9)$ & $18.5(10.6)$ \\
\hline Low $(<12 \%)$ & 27.6 & 24.7 \\
\hline Middle (12-25\%) & 54.0 & 59.0 \\
\hline High $(\geq 25 \%)$ & 18.4 & 16.3 \\
\hline HAQ ${ }^{1}$ Score (0-3) & $0.52(0.65)$ & $0.82(0.70)$ \\
\hline WOMAC ${ }^{2}$ Function (0-68) & $15.6(16.1)$ & $23.3(16.7)$ \\
\hline WOMAC Pain (0-20) & $4.58(4.80)$ & $7.15(4.98)$ \\
\hline WOMAC Total (0-96) & $22.4(22.1)$ & $33.7(22.6)$ \\
\hline
\end{tabular}

$\mathrm{HAQ}=$ Health Assessment Questionnaire

${ }^{2}$ WOMAC $=$ Western Ontario and McMaster Universities Index of Osteoarthritis questionnaire.

${ }^{3} \mathrm{PAS}=$ Physical activity score related to occupation

${ }^{4} \mathrm{NM}=$ non-managerial occupation

Hip rOA analyses. The parameter estimates for adjusted associations between disability scores and SES variables for those with rOA are presented in Table 2. Positive parameter estimates, as all these are, represent increases in disability due to being in the lower SES category in relation to the referent group. The three SES measures most likely contain some of the same information, that is, having a low education may also lead to a non-managerial (physically demanding) job, and to residence in a community of higher poverty rates. In the first set of analyses (Table 2a), the objective was to determine whether the SES variables singly adjusted for covariates have a clear association with disability outcomes with hip rOA.

Low educational attainment was significantly associated with disability as measured by the HAQ and all WOMAC 
scores $(\mathrm{p}<0.05)$. The parameter estimates vary considerably across the outcomes but tend to reflect similar proportions of the underlying ranges for the outcome variables. The three WOMAC scores are also strongly associated $(p<0.01)$ with having a non-managerial occupation, however HAQ is not significantly associated with occupation. Communities with high household poverty rates, compared to the most affluent class of $<12 \%$ are significantly associated with HAQ. Although the parameter estimates are sizable for poverty rates associated with WOMAC function and total, they do not reach significance $(\mathrm{p}=0.054$ and 0.056 , respectively, for medium poverty rate).

The results from multivariable linear regressions of disability and pain measures on all SES variables simultaneously and covariates for hip rOA are displayed in Table 2b. In this second set of analyses (Table 2b), the objective is to see if any of the SES variables have independent associations with disability outcomes and to see which variables may emerge as significant even after adjustment for the others.

When introducing all three SES variables simultaneously as main explanatory variables for disability, low educational attainment remained significantly associated with HAQ $(p<0.01)$. Associations between educational attainment and WOMAC scores remained significant for function and total, but did not remain significant for WOMAC pain. Nonmanagerial (physically demanding) occupation did not remain significant for WOMAC function and WOMAC total but was significant for WOMAC pain $(\mathrm{p}<0.05)$.

Hip symptomatic analyses. The parameter estimates for adjusted associations between disability scores and SES variables for the subgroup with symptomatic OA are presented in Table 3. In the first set of analyses (Table 3a), the objective was to determine the SES variables that have a clear association with pain singly adjusted for covariates and disability outcomes in those with symptomatic OA. Among those in the symptomatic subgroup, low educational attainment has a strong association $(\mathrm{p}<0.01)$ with HAQ and is significantly $(p<0.05)$ associated with WOMAC function and total, but not pain. Occupation is significantly associated with all WOMAC scores $(p<0.05)$ but not HAQ. Finally, community poverty rates are not associated with any disability measures in people with hip symptomatic OA. In the second set of analyses (Table $\mathbf{3 b}$ ), results from multivariable linear regressions of disability and pain measures and all SES variables analyzed simultaneously are displayed. Similar to hip rOA analyses, the objective of this analysis is to see if any of the SES variables have independent associations with symptomatic OA and emerge as significant even after adjustment for the others.

For this symptomatic OA subgroup, educational attainment was the only significant independent SES predictor of HAQ disability. Occupation (non-managerial) is an independent SES predictor associated with WOMAC pain and WOMAC total, but no associations are seen with HAQ or WOMAC function scores.

Considering the known risk factors that were covariates in the models, being female, BMI $>30$, having increased number of comorbidities, or having knee symptoms, generally showed significant, positive associations with HAQ and WOMAC disability measures. Knee symptoms had the largest effect on disability as measured by the magnitudes of the parameter estimates. These findings were as expected.

\section{DISCUSSION}

Our study was designed to confirm and extend observations of associations between measures of individual SES with disability and pain in persons with hip rOA or symptomatic hip OA, while adjusting for known predictors

Table 2. Adjusted ${ }^{1}$ Parameter Estimates for Each of the 3 Socioeconomic Status Variables (Education, Occupation, or Poverty Rate) Singly and Simultaneously Associated with HAQ and WOMAC Health Outcomes ${ }^{2}$ Scores in Persons with Radiographic Hip OA ${ }^{3}$

a. Adjusted ${ }^{1}$ parameter estimates for the 3 socioeconomic variables singly associated with HAQ and WOMAC health outcomes scores.

\begin{tabular}{|c|c|c|c|c|}
\hline Variable & HAQ $^{2}$ (Range 0-3) & WOMAC $^{2}$ Function (Range 0-68) & WOMAC Pain (Range 0-20) & WOMAC Total (Range 0-96) \\
\hline \hline Educational attainment, $<12$ yrs & $0.14^{* *}[0.05,0.24]$ & $4.07^{* *}[1.76,6.37]$ & $0.90^{*}[0.22,1.58]$ & $5.19^{* *}[2.05,8.34]$ \\
\hline Occupation, $\mathbf{N M}^{4}$ & $0.03[-0.06,0.11]$ & $3.13^{* *}[0.95,5.31]$ & $0.98^{* *}[0.34,1.63]$ & $4.15^{* *}[1.17,7.13]$ \\
\hline Poverty Rate, Medium $(12-25 \%)$ & $0.09[0,0.19]$ & $2.41[-0.04,4.86]$ & $0.53[-0.2,1.25]$ & $3.24[-0.09,6.57]$ \\
\hline Poverty Rate, High $(\geq 25 \%)$ & $0.15^{*}[0.01,0.29]$ & $2.15[-1.33,5.63]$ & $0.65[-0.38,1.68]$ & $3.01[-1.73,7.75]$ \\
\hline
\end{tabular}

b Adjusted ${ }^{1}$ parameter estimates for all 3 socioeconomic variables simultaneously associated with HAQ and WOMAC health outcomes scores

\begin{tabular}{|c|c|c|c|c|}
\hline \hline Educational attainment, $<12 \mathrm{yrs}$ & $0.15^{* *}[0.05,0.35]$ & $3.22^{*}[0.73,5.7]$ & $0.57[-0.16,1.3]$ & $4.01 *[0.63,7.4]$ \\
\hline Occupation, $\mathbf{N M}^{4}$ & $-0.03[-0.12,0.07]$ & $2.01[-0.33,4.34]$ & $0.78 *[0.09,1.47]$ & $2.72[-0.47,5.91]$ \\
\hline Poverty Rate, Medium $(12-25 \%)$ & $0.09[-0.01,0.19]$ & $2.31[-0.12,4.74]$ & $0.49[-0.23,1.21]$ & $3.09[-0.21,6.4]$ \\
\hline Poverty Rate, High $(\geq 25 \%)$ & $0.13[-0.01,0.27]$ & $1.80[-1.66,5.26]$ & $0.58[-0.44,1.61]$ & $2.56[-2.16,7.28]$ \\
\hline
\end{tabular}

\footnotetext{
$* * \mathrm{p}<0.01 ; * \mathrm{p}<0.05$.
}

${ }^{1}$ All models are adjusted for age, gender, BMI, race, knee symptoms, comorbidity, occupational physical activity score.

${ }^{2} \mathrm{HAQ}=$ Health Assessment Questionnaire, $\mathrm{n}=708$, WOMAC $=$ Western Ontario and McMaster Universities Osteoarthritis Index, $\mathrm{n}=688$.

${ }^{3}$ Values are the $\beta[95 \%$ confidence interval $]$.

${ }^{4} \mathrm{NM}=$ non-managerial, physically demanding. 
Table 3. Adjusted ${ }^{1}$ Parameter Estimates for Each of the 3 Socioeconomic Status Variables (Education, Occupation, or Poverty Rate) Singly and Simultaneously Associated with HAQ and WOMAC Health Outcomes ${ }^{2}$ Scores in Persons with Symptomatic Hip $\mathbf{O A}^{3}$

\begin{tabular}{|c|c|c|c|c|}
\hline a Adjusted parameter estimates for the 3 socioeconomic variables singly associated with HAQ and WOMAC health outcomes scores. \\
\hline Variable & HAQ ${ }^{2}$ (Range 0-3) & WOMAC ${ }^{2}$ Function (Range 0-68) & WOMAC Pain (Range 0-20) & WOMAC Total (Range 0-96) \\
\hline \hline Educational attainment, $<12$ yrs & $0.25^{* *}[0.09,0.41]$ & $4.5^{*}[0.48,8.51]$ & $0.90[-0.32,2.12]$ & $5.66^{*}[0.2,11.1]$ \\
\hline Occupation, NM ${ }^{4}$ & $0.10[-0.06,0.27]$ & $5.12^{*}[1.05,9.19]$ & $1.72 * *[0.48,2.96]$ & $7.40^{* *}[1.87,12.9]$ \\
\hline Poverty Rate, Medium $(12-25 \%)$ & $0.06[-0.12,0.24]$ & $1.38[-3.19,5.96]$ & $0.08[-1.31,1.47]$ & $1.66[-4.56,7.88]$ \\
\hline Poverty Rate, High $(\geq 25 \%)$ & $0.06[-0.21,0.32]$ & $-0.12[-6.71,6.48]$ & $0.31[-1.69,2.32]$ & $0.29[-8.67,9.26]$ \\
\hline b Adjusted parameter estimates for all 3 socioeconomic variables simultaneously associated with HAQ and WOMAC health outcomes scores \\
\hline \hline Educational attainment, $<12$ yrs & $0.24 * *[0.07,0.41]$ & $3.17[-1.10,7.44]$ & $0.33[-0.97,1.63]$ & $3.56[-2.24,9.37]$ \\
\hline \multicolumn{6}{|c|}{ Occupation, NM ${ }^{4}$} & $0.02[-0.16,0.19]$ & $4.02[-0.32,8.37]$ & $1.61 *[0.28,2.95]$ & $6.16^{*}[0.23,12.1]$ \\
\hline Poverty Rate, Medium $(12-25 \%)$ & $0.05[-0.13,0.24]$ & $1.48[-3.04,6.00]$ & $0.09[-1.29,1.46]$ & $1.73[-4.41,7.87]$ \\
\hline Poverty Rate, High $(\geq 25 \%)$ & $0.04[-0.22,0.30]$ & $0.10[-6.45,6.64]$ & $0.42[-1.57,2.41]$ & $0.57[-8.31,9.45]$ \\
\hline
\end{tabular}

$* * \mathrm{p}<0.01 ; * \mathrm{p}<0.05$.

${ }^{1}$ All models are adjusted for age, gender, BMI, race, knee symptoms, comorbidity, occupational physical activity score.

${ }^{2} \mathrm{HAQ}=$ Health Assessment Questionnaire, $\mathrm{n}=251$, WOMAC $=$ Western Ontario and McMaster Universities Osteoarthritis Index, $\mathrm{n}=244$

${ }^{3}$ Values are the $\beta[95 \%$ confidence interval].

${ }^{4} \mathrm{NM}=$ non-managerial, physically demanding.

of disability. In addition, we examined the associations of community SES with disability in our sample of 708 African American and Caucasian Johnston County residents with hip rOA, of whom 251 also had symptoms in the same hip that had rOA. Individuals with less than 12 years of schooling were more likely to have significantly higher HAQ and WOMAC scores, reflecting more disability and pain, than those with higher educational attainment, even after adjusting for age, gender, BMI, race, occupational physical activity score, comorbidities, and the presence of knee symptoms

Significant associations were also noted between individuals with non-managerial occupations and higher WOMAC scores, but not with HAQ scores, in participants with hip rOA or the subgroup with hip symptomatic OA. In participants with rOA, residing in a Census block group with a community poverty rate of $25 \%$ or greater was associated with increased disability, measured by the HAQ, although the magnitude of these findings were weaker than for those with low educational attainment.

In multivariable analyses introducing all three SES variables simultaneously as main explanatory variables for disability, educational attainment was the only significant independent SES predictor of HAQ disability in both the rOA group and symptomatic OA subgroup and of WOMAC function and WOMAC total in rOA. However, the parameter estimate for the association between education and WOMAC function in the symptomatic hip OA sample is sizable; the small sample size $(n=244)$ may make the determination of significance more difficult. Occupation was the only significant SES predictor of WOMAC pain in both radiographic and symptomatic hip OA.

Looking at magnitudes of the parameter estimates across Tables $\mathbf{2}$ and 3, without regard to the significance, it may be that education is most associated with HAQ and WOMAC function, whereas occupation tends to be stronger in its association with WOMAC pain and WOMAC total. In rOA alone, living in a community with the highest poverty rate tends to be associated with HAQ.

As would be expected, variables that are known to be associated with disability and pain in hip OA, including gender, BMI and knee symptoms were strongly associated with disability in our population with hip OA. However, even after adjusting for these covariates, there was still a significant association of educational attainment with HAQ scores and educational attainment and occupation with WOMAC scores.

Our study of a Caucasian and African American population in the United States confirmed findings from the three European studies identifying educational attainment as a significant factor associated with WOMAC function in individuals with hip OA [14-16]. In those studies, educational attainment was not associated with hip pain, but we extended the work of these European studies to examine other SES variables, occupation and community poverty, with WOMAC. Interestingly, occupation was linked significantly with WOMAC pain but not function for persons with both rOA and symptomatic OA in the multivariable analyses. We also included another commonly used measure of arthritis disability, the HAQ. Our study, like the Finnish study [14], included knee symptoms which, as stated earlier, involve pain, aching or stiffness that could ultimately be responsible for a participant's measured disability even if that participant exhibited hip rOA or symptomatic OA. The knee symptoms were a strong contributor to disability, but even after controlling for these symptoms and other important risk factors, SES variables contributed significantly. Our study was also the first study to include an area-level measure of SES, community poverty, in addition to individual level SES measures, education and occupation. Measuring community SES by community poverty level 
may be rather non-specific, but it is an all-encompassing single variable that may represent both the physical community environment as well as the physical and mental health of its residents. For example, this could include poorer access to exercise facilities, public transportation, or medical care, as well as fewer health-conscious neighbors or residents of the same household.

The strengths of this investigation lie in its welldescribed bi-racial study population of men and women, its inclusion of both persons with radiographic and symptomatic OA instead of only persons with hip pain or other symptoms when considering disability, as well as its adjustment for knee symptoms. The study population was also enriched with groups at higher risk for OA outcomes and had a high rate of participation. All participants underwent identical clinical and in-home interviews. We expect that these estimates will generalize to our target population of civilian, non-institutionalized, African Americans or Caucasians, aged 45 years or older, residing in a rural community.

Additionally, this study uses a population in the United States, as opposed to similar studies that took place in Europe and entailed a remarkably different patient population; the populations in these European studies are cared for under a different healthcare system than in the United States. The limitations of our study include the lack of other potentially important variables of individual SES, such as income, and the crude measure of community SES we are using by employing Census block group poverty level. Although it would be desirable to include other measures, these measures are ones that are easily obtainable and appear to give a good reflection of both individual and community SES.

\section{CONCLUSION}

This study highlights the importance of understanding a person's socioeconomic standing, everyday life and physical environment when treating hip $\mathrm{OA}$ and the clinicallysignificant influences of SES on disability. These data underscore the importance of individual SES variables above and beyond other contributors to disability outcomes in hip OA. These data also underscore the importance of a clinician acknowledging the contribution of factors outside the medical system to health status.

\section{ACKNOWLEDGMENTS}

The authors thank the staff of the Johnston County Osteoarthritis Project for their long-standing and dedicated work, as well as the Project participants, without whose continuing cooperation none of this work would be possible.

\section{FUNDING SOURCES}

Funding for this project was provided by the American College of Rheumatology Research and Education Foundation Award; the National Institute of Arthritis and Musculoskeletal Skin Diseases (NIAMS) grant numbers: RO1-AR-053-989-01, 5-P60-AR-30701, and 5-P60AR49465; and cooperative agreements S043 and S3486 from the Centers for Disease Control and Prevention through the Association of Schools of Public Health.

\section{CONFLICT OF INTEREST}

None of the authors have financial or personal relationships to disclose. No funds were provided for writing this manuscript.

\section{REFERENCES}

[1] Jordan KP, Thomas E, Peat G, Wilkie R, Croft P. Social risks for disabling pain in older people: a prospective study of individual and area characteristics. Pain 2008; 137(3): 652-61.

[2] Hootman JM, Helmick CG, Schappert SM. Magnitude and Characteristics of Arthritis and Other Rheumatic Conditions on Ambulatory Medical Care Visits, United States, 1997. Arthritis \& Rheumatism 2002; 47(6): 571-81.

[3] Buckwalter JA, Saltzman C, Brown T. The impact of osteoarthritis: implications for research. Clin Orthop Relat Res 2004; (Suppl 427): S6-15.

[4] Gabriel SE, Crowson CS, Campion ME, O'Fallon WM. Direct medical costs unique to people with arthritis. J Rheumatol 1997; 24(4): 719-25.

[5] Paans N, van dA-S, I, van der MK, Bulstra SK, Stevens M. The effects of exercise and weight loss in overweight patients with hip osteoarthritis: design of a prospective cohort study. BMC Musculoskelet Disord 2009; 10: 24.

[6] Jordan JM, Helmick C, Renner JB, et al. Prevalence of hip symptoms and radiographic and symptomatic hip osteoarthritis in African-Americans and Caucasians: The Johnston County Osteoarthritis Project. J Rheumatol 2009; 36(4): 809-15.

[7] Jordan JM, Callahan LF. Community and Environmental Factors and Arthritis Outcomes. N C Med J 2007; 68(6): 439-43.

[8] Fernandez-Lopez JC, Laffon A, Blanco FJ, Carmona L, EPISER Study Group. Prevalence, risk factors, and impact of knee pain suggesting osteoarthritis in Spain. Clin Exp Rheumatol 2008; 26(2): 324-32.

[9] Felson DT, Hannan MT, Naimark A, et al. Occupational physical demands, knee bending, and knee osteoarthritis: results from the Framingham Study. J Rheumatol 1991; 18(10): 1587-92.

[10] Holte HH, Tambs K, Bjerkedal T. Manual work as predictor for disability pensioning with osteoarthritis among the employed in Norway 1971-1990. Int J Epidemiol 2000; 29(3): 487-94.

[11] Sainio P, Martelin T, Koskinen S, Heliovaara M. Educational differences in mobility: the contribution of physical workload, obesity, smoking and chronic conditions. J Epidemiol Community Health 2007; 61(5): 401-8.

[12] Callahan LF, Smith WJ, Pincus T. Self-report questionnaires in five rheumatic diseases: comparisons of health status constructs and associations with formal education level. Arthritis Care Res 1989; 2(4): 122-31.

[13] Abraido-Lanza AF, White K, Armbrister AN, Link BG. Health status, activity limitations, and disability in work and housework among Latinos and non-Latinos with arthritis: an analysis of national data. Arthritis Rheum 2006; 55(3): 442-50.

[14] Juhakoski R, Tenhonen S, Anttonen T, Kauppinen T, Arokoski JP. Factors affecting self-reported pain and physical function in patients with hip osteoarthritis. Arch Phys Med Rehabil 2008; 89(6): 1066-73.

[15] Dieppe P, Judge A, Williams S, et al. Variations in the preoperative status of patients coming to primary hip replacement for osteoarthritis in European orthopaedic centres. BMC Musculoskelet Disord 2009; 10: 19.

[16] Schafer T, Krummenauer F, Mettelsiefen J, Kirschner S, Gnnther KP. Social, educational, and occupational predictors of total hip replacement outcome. Osteoarthritis Cartilage 2010.

[17] Yong PF, Milner PC, Payne JN, Lewis PA, Jennison C. Inequalities in access to knee joint replacements for people in need. Ann Rheum Dis 2004; 63(11): 1483-9.

[18] Martin SA, Haren MT, Taylor AW, Middleton SM, Wittert GA. Florey Adelaide Male Ageing Study (FAMAS). Chronic disease prevalence and associations in a cohort of Australian men: the Florey Adelaide Male Ageing Study (FAMAS). BMC Public Health 2008; 8: 261.

[19] Chacon JG, Gonzalez NE, Veliz A, et al. Effect of knee osteoarthritis on the perception of quality of life in Venezuelan patients. Arthritis Rheum 2004; 51(3): 377-82. 
[20] Wilkie R, Peat G, Thomas E, Croft P. Factors associated with restricted mobility outside the home in community-dwelling adults ages fifty years and older with knee pain: An example of use of the International Classification of Functioning to investigate participation restriction. Arthritis Care \& Research 2007; 57(8): $1381-9$.

[21] Keysor JJ, Jette AM, LaValley MP, et al. Community environmental factors are associated with disability in older adults with functional limitations: the MOST study. J Gerontol A Biol Sci Med Sci 2010; 65(4): 393-9.

[22] Jordan JM, Helmick CG, Renner JB, et al. Prevalence of knee symptoms and radiographic and symptomatic knee osteoarthritis in African-Americans and Caucasians: The Johnston County Osteoarthritis Project. J Rheumatol 2007; 34(1): 172-80.

[23] Murphy L, Schwartz TA, Helmick CG, et al. Lifetime Risk of Symptomatic Knee Osteoarthritis. Arthritis Rheum 2008; 59(9): 1207-13.

[24] Kellgren JH, Lawrence JS. Radiological assessment of osteoarthrosis. Ann Rheum Dis 1957; 16(4): 494-502.

[25] Jordan JM, Linder GF, Renner JB, Fryer JG. The impact of arthritis in rural populations. Arthritis Care Res 1995 Dec; 8(4): 242-50.

[26] Callahan L, Shreffler J, Siaton B, et al. Limited educational attainment and radiographic and symptomatic knee osteoarthritis: a cross-sectional analysis using data from the Johnston County (North Carolina) Osteoarthritis Project. Arthritis Res Ther 2010; 12(2): R46.

[27] Fries JF, Spitz P, Kraines RG, Holman HR. Measurement of patient outcome in arthritis. Arthritis Rheum 1980; 23(2): 137-45.

[28] Bellamy N. WOMAC osteoarthritis index: a user's manual. London, Ontario: Dr. Nicholas Bellamy; 1996.
[29] Bruce B, Fries J. Longitudinal comparison of the Health Assessment Questionnaire (HAQ) and the Western Ontario and McMaster Universities Osteoarthritis Index (WOMAC). Arthritis Care Res 2004; 51(5): 730-7.

[30] Bellamy N, Buchanan WW, Goldsmith CH, Campbell J, Stitt L. Validation study of the WOMAC: a health status instrument for measuring clinically-important patient relevant outcomes following total hip or knee arthroplasty in osteoarthritis. J Orthoped Rheumatol 1997; 1: 95-108.

[31] McConnell S, Kolopack P, Davis AM. The Western Ontario and McMaster Universities Osteoarthritis Index (WOMAC): A Review of Its Utility and Measurement Properties. Arthritis Care Res 2011; 45(5): 453-61.

[32] US Department of Commerce - Bureau of the Census. 1990 census of population and housing. Washington: US GPO; 1990.

[33] Gomez-Marin O, Fleming LE, Caban A, LeBlanc WG, Lee DJ, Pitman T. Longest Held Job in U.S. Ocuupational Groups: The National Health Interview Survey. J Occup Environ Med 2005; 47(1): 79-89.

[34] Bureau of the Census U. Census of Population and Housing, Census 2000 Summary File 3. 2002. Washington, DC, Department of Commerce.

[35] U.S.Bureau of the Census. 2000 Census. U S Census Bureau, the Official Statistics 2010 June 28 [cited 2002 Jun 5]; Available from: URL: http: //www.census.gov

[36] Allen KD, Helmick CG, Schwartz TA, DeVellis RF, Renner JB, Jordan JM. Racial differences in self-reported pain and function among individuals with radiographic hip and knee osteoarthritis: the Johnston County Osteoarthritis Project. Osteoarthritis Cartilage $2009 ; 17(9): 1132-6$

This is an open access article licensed under the terms of the Creative Commons Attribution Non-Commercial License (http://creativecommons.org/licenses/by-nc/ 3.0/) which permits unrestricted, non-commercial use, distribution and reproduction in any medium, provided the work is properly cited. 